\title{
Indicadores ecológicos y herramientas para la gestión de ecosistemas acuáticos en la zona costera
}

\author{
F.A. Comín ${ }^{1}$, M. Menéndez ${ }^{1}$, J.A. Romeroํㅜ, O. Hernández ${ }^{1}$, M. Martínez ${ }^{1}$, A. Chacón² \\ ${ }^{1}$ Departamento de Ecología. Universidad de Barcelona. Diagonal 645. 08028. Barcelona. España. ${ }^{2}$ Universidad \\ de Chapingo, Chapingo. México.
}

\section{RESUMEN}

La gestión de las zonas costeras debería producir resultados a corto plazo en la cualidad ambiental y en la conservación de los hábitats a largo plazo. Los cambios en las características del agua y en la importancia de los productores primarios que tuvieron lugar en una laguna costera (Tancada) del Delta del Ebro (NE España) durante la última década, son buenos indicadores de la conservación de la zona costera. Durante los periodos de bajo flujo de entrada de agua dulce, ocurren elevadas concentraciones de amonio (3.2-44 mmol L-1), alta conductividad (32-61 $\mathrm{mS} \mathrm{cm}^{-1}$ ), y baja biomasa (máximo de $57.6 \mathrm{~g} \mathrm{PS} \mathrm{m}^{-2}$ ) y cobertura de macrófitos sumergidos enraizados (aproximadamente un 10\% del área de la laguna) así como proliferación de macroalgas. Durante periodos con un flujo de entrada relativamente elevado, ocurren bajas concentraciones de amonio (0-20 mmol L-1), conductividad intermedia $\left(10-45 \mathrm{mS} \mathrm{cm}^{-1}\right.$ ), elevadas biomasa (máximo $579 \mathrm{~g} \mathrm{PS} \mathrm{m}^{-2}$ ) y cobertura de macrófitos sumergidos enraizados (50-75\%) y ausencia de proliferación de macroalgas. La conservación de la zona costera debería basarse en la manutención de los flujos naturales de agua entre el mar y el continente, o en la estimulación del papel de ecosistemas naturales como zonas para tamponar los impactos de usos humanos intensivos originados por las descargas de aguas, impactando negativamente en los ecosistemas naturales. Las zonas húmedas recuperadas en la vecindad de la laguna de Tancada retienen casi el 100\% del nitrógeno entrante y, durante algunos periodos, una gran parte del fósforo descargado con los residuos agrícolas. La recuperación de las zonas húmedas que conduzca al incremento de la heterogeneidad espacial de los habitats es un objetivo a largo plazo que debe incorporar criterios geomorfológicos. En el área de estudio, las actividades antropogénicas disminuyeron la biodiversidad del hábitat durante 1970-1992. Las recientes actividades de restauración de las zonas húmedas demuestran que es posible recuperar esta diversidad de hábitat, lo que puede ser una herramienta importante para la gestión de la zona costera.

Palabras clave: zona costera, calidad del agua, biodiversidad, manejo.

\begin{abstract}
Management of the coastal zone should achieve goals of environmental quality in the short term and conservation of habitats in the long term. Changes in water quality and in the importance of primary producers, taking place in a coastal lagoon (Tancada) of the Ebro Delta (NE Spain) during the last decade, are good indicators of the conservation of the coastal zone. During periods of low freshwater inflows, high concentrations of ammonium (3.2-44 $\mu$ mol L-1), high conductivity (32-61mS $\mathrm{cm}^{-1}$ ), low biomass (maximum of $57.6 \mathrm{~g} \mathrm{DW} \mathrm{m}^{-2}$ ), low coverage of submerged rooted macrophytes (i.e. $10 \%$ of the lagoon area) and macroalgae blooms occur. By contrast during periods with relatively high water flows, wetlands present concentrations of ammonium (0-20 $\left.\mathrm{mmol} \mathrm{L}^{-1}\right)$, intermediate conductivity levels $\left(10-45 \mathrm{mS} \mathrm{cm}^{-1}\right)$, large biomass (maximum $\left.579 \mathrm{~g} \mathrm{DW} \mathrm{m}^{-2}\right)$, and large coverage of submerged rooted macrophytes (50-75\%). In addition, no macroalgal blooming is observed. Coastal-zone conservation should be based on the maintenance of the natural water flows between the sea and mainland. Alternatively, stimulate of the role of natural ecosystems as buffering zones of human impact, specially waste water discharge. Restored wetlands in the vicinity of Tancada lagoon retained almost $100 \%$ of the inflowing nitrogen and, during some periods, a large part of the phosphorus discharged with agricultural sewage. Wetland restoration addressed to increasing spatial heterogeneity of habitats is a long-term challenge which must incorporate geomorphological criteria. In the study area, human impact
\end{abstract}

Limnetica 16: 61-68 (1999)

(C) Asociación Española de Limnología, Madrid. Spain. ISSN: 0213-8409. 
decreased habitat diversity during 1970-1992. Recent wetland restoration activities demonstrate that it is possible to recover this habitat diversity, and that it could be an important tool for the management of the coastal zone.

Key words: coastal zone, water quality, biodiversity, management.

\section{INTRODUCCIÓN}

La gestión de la zona costera con la finalidad de que sus recursos naturales se mantengan de forma perdurable debería realizarse con objetivos claros de calidad ambiental a corto plazo y de preservación territorial a largo plazo. Para ello se requiere información simple del estado de los ecosistemas y de herramientas, a ser posible, sencillas y baratas que hagan variar esa calidad ambiental en la dirección deseada.

En general, los ecosistemas de la zona costera se caracterizan, en comparación con ecosistemas de otras zonas geográficas, por un intenso dinamismo. Sus características - en un momento concreto - pueden ser estadios transicionales dentro de una situación variable o de equilibrio inestable. Reconocer la dimensión de esta escala de variación y los diversos estados de transición ayuda a diagnosticar la situación ambiental y a definir los objetivos de gestión de la zona costera.

En este trabajo se presentan indicadores ecológicos que sintetizan conjuntos de variables para definir la situación ambiental de humedales costeros. También se presentan herramientas ecológicas sencillas para manejar los ecosistemas y mejorar su calidad ambiental. Todo ello utilizando datos y experiencias relativas a una zona costera del Delta del Ebro (España) (Fig. 1) (Comín et al., 1995).

\section{MATERIAL Y MÉTODOS}

Se efectuaron medidas in situ de conductividad y temperatura y se muestreó la columna de agua $(n=3)$ para estimar la concentración de nitrógeno inorgánico y fósforo disuelto. Se tomaron muestras de agua en tres zonas húmedas restauradas litorales de la laguna. Se utilizaron métodos estándar para cuantificar las concentraciones de nutrientes disueltos y el nitrógeno total (Strickland \& Parsons, 1965; Koroleff, 1983; APHA et al., 1992). Aquí se presenta una síntesis de los resultados obtenidos en varios trabajos realizados en la misma zona de estudio (Comín et al., 1997, 1998a, 1998b). Los muestreos de biomasa de macrófitos se efectuaron a partir de la metodología descrita en Menéndez \& Comín (1989).

Las superficies ocupadas por cada hábitat en los años 1971, 1983 y 1993 fueron calculadas a partir de fotografia aèrea y ortofotomapas a diferente escala $(1: 5000,1: 18000$ y 1:25000) con la utilización de un planímetro electrónico y corrigiendo las diferencias de escala.

\section{RESULTADOS}

\section{Indicadores ecológicos}

El espacio definido por los ejes de temperatura y salinidad, o un equivalente suyo como la conduc-

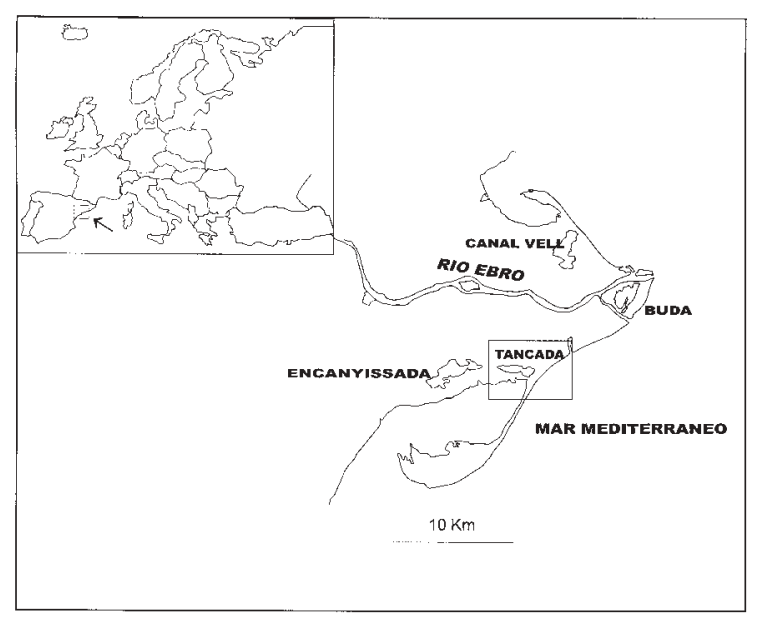

Figura 1. Mapa del Delta del Ebro (España) y de la zona de estudio. Map of the Ebro Delta (Spain) and of the study area. 


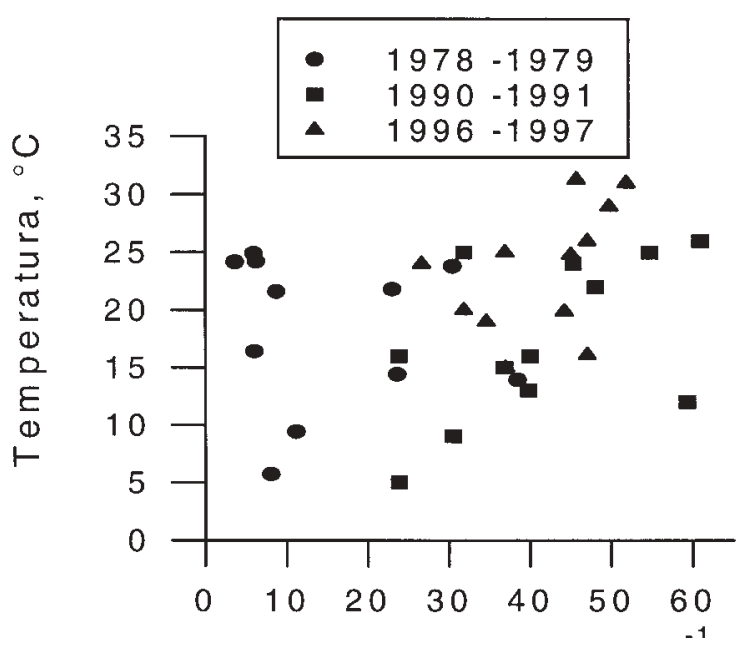

Figura 2. Gráficos temperatura-conductividad del agua en la cubeta Este de la laguna de la Tancada durante periodos de diferente régimen hidrológico. Temperature and conductivity in the west basin of the Tancada lagoon under different hydrological regimes.
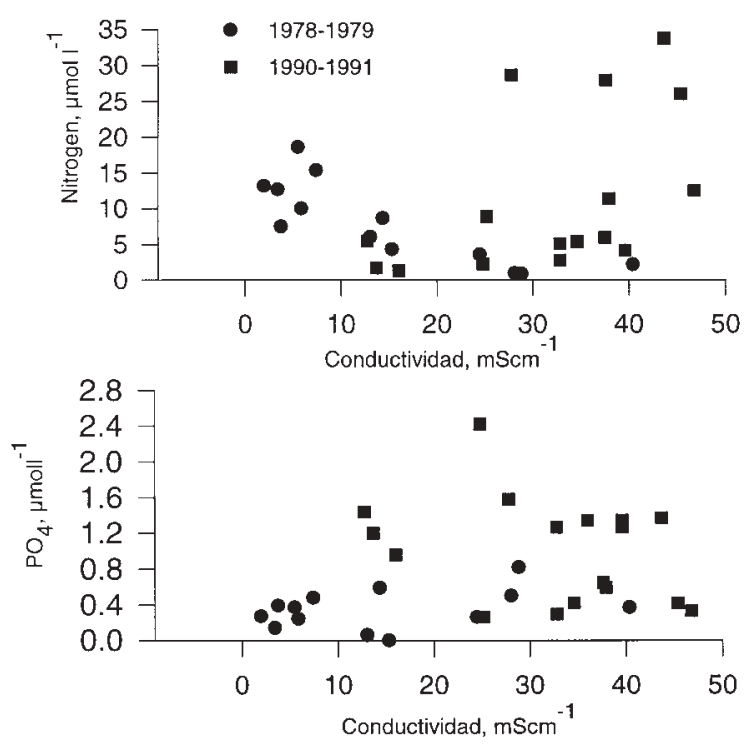

Figura 3. Variaciones de las concentraciones de nitrógeno y fósforo inorgánico disueltos en la laguna de La Tancada. Variations in dissolved nitrogen and inorganic phosphorus concentrations in the Tancada lagoon. tividad, sirve para definir la dinámica temporal de las condiciones ambientales generales en aguas de la zona costera sometidas a influencias de agua dulce y/o de agua marina. En la figura 2 se puede observar que la cubeta $\mathrm{E}$ de la laguna $\mathrm{La}$ Tancada experimentó un episodio de hipersalinidad (salinidad del agua superior a la del mar, 55 $\mathrm{mS} \mathrm{cm}{ }^{-1}$, motivada por concentración salina debida a evaporación superior al efecto potencial de dilución debido al aporte de agua dulce) durante los meses de mayores temperaturas del periodo 1990-91. Es decir, que la laguna experimentó a partir de 1990 un incremento general de la salinidad, debido a la disminución de los aportes de agua dulce por los canales y retornó durante 1996-97 a una dinámica de mezcla más similar a la existente durante épocas anteriores, finales de los años setenta y década de los ochenta (Menéndez \& Comín, 1989; Comín et al., 1991).

Estos cambios hidrológicos han tenido repercusiones en las concentraciones de nutrientes disueltos en el agua. Se ha observado un incremento de las concentraciones de nitrógeno (nitrato más nitrito y amonio) en la parte Este y en los momentos más salados de la laguna durante el periodo 1990-91, es decir, asociados a una menor renovación del agua por mezcla con agua dulce. En cambio, durante periodos o años de notables aportes de agua dulce las mayores concentraciones de este micronutriente están asociadas a estas descargas de agua dulce (Fig. 3). También se observa que la concentración de fosfatos correspondiente a la fracción inorgánica soluble aumenta y permanece mayor durante periodos de menor renovación del agua (1990-91) que durante periodos de mayor renovación (1978-79).

La Tabla 1 resume los cambios más inmediatos de carácter biológico desencadenados por la variación hidrológica consistente en disminuir la renovación del agua de la laguna o aumentarla con entradas de agua dulce o con entradas de agua de mar. Se produce un cambio en la estructura de la producción primaria, disminuyendo la contribución del fitoplancton (concentraciones de pigmentos inferiores a $5 \mathrm{mg} \mathrm{m}^{-3}$ ) y la cobertura de macrófitos sumergidos enraizados, aumentando la cobertura de las macroalgas. En este proce- 
Table 1. Cambios más inmediatos de carácter biológico desencadenados por la variación hidrológica consistente en disminuir la renovación del agua de la laguna o aumentarla con entradas de agua dulce o con entradas de agua de mar ( $\mathrm{r}$ - tasa de renovación). Short-term biological changes produced by hydrological manipulation. In some cases turnover time is reduced while in others it is increased introducing freshwater or seawater ( $r$ - turnover time).

\begin{tabular}{|c|c|c|c|}
\hline & $\mathrm{r} \uparrow$ con agua dulce. & $\mathrm{r} \downarrow$ & $\mathrm{r} \uparrow$ con agua de mar \\
\hline $\begin{array}{l}\text { Pigmentos. Fitoplàncton } \\
\mathrm{mg} \mathrm{m}^{-3}\end{array}$ & 43.09 & 1.43 & $5.62-9.02$ \\
\hline $\begin{array}{l}\text { Cobertura, \% } \\
\text { mac. enraizados }\end{array}$ & 80 & 5 & 60 \\
\hline $\begin{array}{l}\text { Biomasa max. } \\
\text { mac. enrraizados } \\
\text { g PS m-2 }\end{array}$ & $\begin{array}{c}617 \\
\text { R.cirrhosa }\end{array}$ & $\begin{array}{c}57.6 \\
\text { R.cirrhosa }\end{array}$ & --- \\
\hline $\begin{array}{l}\text { Cobertura, \% } \\
\text { Macroalgas }\end{array}$ & $\begin{array}{c}5 \\
\text { Chaetomorpha } \\
\text { Cladophora }\end{array}$ & $\begin{array}{c}40 \\
\text { Chaetomorpha } \\
\text { Cladophora } \\
\text { Ulva }\end{array}$ & $\begin{array}{c}20 \\
\text { Chaetomorpha } \\
\text { Cladophora } \\
\text { Ulva } \\
\text { Chondria Gracilaria }\end{array}$ \\
\hline $\begin{array}{l}\text { Biomasa max. } \\
\text { Macroalgas } \\
\text { g PS m}^{-2}\end{array}$ & --- & 250 & 665 \\
\hline
\end{tabular}

so dinámico, a lo largo de varios años, tiene lugar una relativa estabilización de la distribución de la producción primaria a favor de las macroalgas (en términos de biomasa) con una relativa recuperación (hasta ahora solo comprobado en términos de cobertura) de los macrófitos sumergidos enraizados. Además tiene lugar una colonización de la laguna por mayor número de especies adaptadas a las condiciones ambientales impuestas por el nuevo régimen hidrológico.

\section{Herramientas de gestión}

La figura 4 muestra los cambios en la salinidad media y las variaciones de la salinidad, ambas relativas, de la laguna La Tancada durante los últimos años. En ella está representada la situación ideal para ambientes acuáticos costeros bajo distinto régimen hidrológico. Esta figura proporciona un marco de referencia orientativo para marcar los objetivos a los cuales se debe dirigir la gestión de

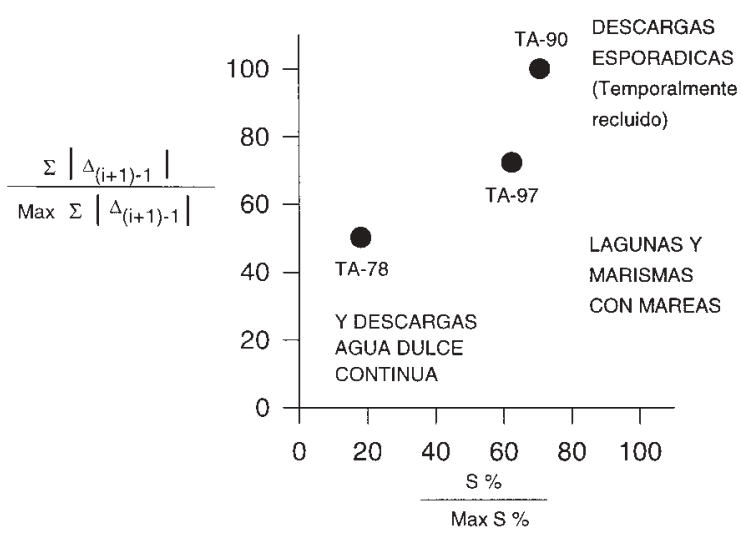

Figura 4. Situación de la laguna La Tancada en cuanto a su salinidad media relativa (eje horizontal) y a las variaciones relativas de salinidad durante periodos de régimen hidrológico diferente, que muestran su variación a lo largo de los últimos años. Se indica también la situación ideal para lagunas bajo distinto régimen hidrológico. Average relative salinity (horizontal axis) and variations in salinity under different hydrological regimes recently experienced by the Tancada lagoon. Ideal situations for lagoons under different hydrological regimes are also indicated. 
una laguna costera, como ejemplo de ambiente acuático salobre. Según cual sea el objetivo deseado, se requerirá mayor o menor renovación del agua de la laguna con agua dulce o marina.

La figura 5 resume los resultados de años de observación en la dominancia relativa de la producción primaria en la laguna La Tancada (Menéndez \& Comín, 1989; Comín et al., 1990). Todo cambio hidrológico representa un impacto en las poblaciones asentadas en la laguna que puede ser positivo o negativo para ellas y una colonización por parte de otras especies que pueden llegar a desplazar a las establecidas previamente, como es el caso de la proliferación de macroalgas si se llega de nuevo a un régimen de variación de la salinidad del agua dentro de rangos elevados, incluso de hipersalinidad, y con variaciones temporales intensas de la concentración disponible en el agua de nitrógeno inorgánico disuelto. En otras condiciones de relativa constancia de la salinidad, pueden llegar a establecerse praderas extensas de macrófitos sumergidos enraizados (Ruppia cirrhosa si la salinidad es alta y Potamogeton pectinatus si la salinidad es baja). La dominancia de estas poblaciones tarda un tiempo debido a su menor tasa de crecimiento comparada con otros productores primarios de crecimiento poblacional más rápido, por ejemplo fitoplancton, sobre todo si existen abundantes elementos nutritivos disponibles en el agua.

El establecimiento, en los márgenes de la laguna, de áreas con macrófitos emergentes por las cuales pase el agua de entrada a la laguna, es una excelente herramienta para reducir la entrada de elementos nutritivos, porque la eficiencia de retención de nutrientes es muy alta en este tipo de sistemas ecológicos (Tabla 2). Se llega a retener hasta el 99\% del nitrógeno inorgánico disuelto que entra a estas áreas y una porción variable a lo largo del tiempo del fósforo. Debido a la liberación de fósforo que tiene lugar del sedimento a la columna de agua, en ocasiones salen concentraciones mayores de fósforo que entran a estos sistemas, variando su eficiencia de retención en función de las cargas de entrada y del régimen hidrológico (Comín et al., 1997, 1998a, 1998b).

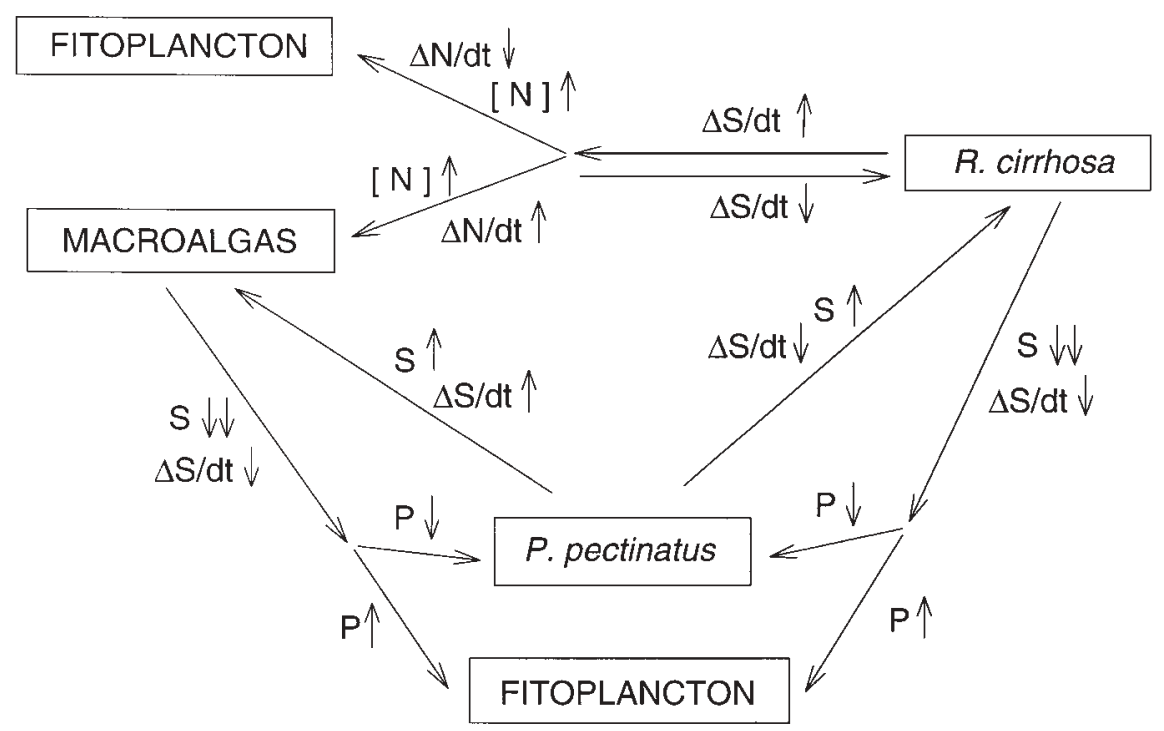

Figura 5. Cambios en la dominancia de los productores primarios de ambientes acuáticos costeros en función de las variaciones de salinidad y micronutrientes principales. (Las flechas indican aumento o disminución. El triángulo indica variación en función del tiempo). Changes in dominance of primary producers in relation to variations in salinity and main micronutrients. Arrows indicate increase or decrease. The triangle indicates variations. 
Table 2. Eficiencias en la retención de nutrientes de tres humedales restaurados (media \pm SD) de 50 x $150 \mathrm{~m}$ situados en el margen de la laguna y dominados por Phragmites australis. Nutrient retention efficiency in three $50 \times 150 \mathrm{~m}$ restored wetlands (mean \pm 1 SD) placed on the shore of the lagoon and dominated by Phragmites australis.

\begin{tabular}{lrrr}
\hline & $\begin{array}{c}\text { Concentración Entrada } \\
\mathbf{m g} / \mathbf{m}^{2} / \mathbf{d i ́ a}\end{array}$ & $\begin{array}{c}\text { Concentración } \\
\text { Salidamg/m²/día }\end{array}$ & Eficiencia \% \\
\hline $\mathbf{N}-\left(\mathbf{N O}_{3}{ }^{-}+\mathbf{N O}_{2}{ }^{-}\right)$ & $41,76 \pm 25,17$ & $0,14 \pm 0,16$ & $99,59 \pm 0,56$ \\
$\mathbf{N}^{+} \mathbf{N H}_{4}^{+}$ & $2,33 \pm 2,35$ & $0,85 \pm 0,60$ & $31,71 \pm 84,40$ \\
SRP & $2,54 \pm 1,18$ & $2,27 \pm 1,44$ & $-4,29 \pm 84,85$ \\
NIT. TOTAL & $102,73 \pm 77,88$ & $20,75 \pm 16,11$ & $74,22 \pm 16,26$ \\
DIN & $63,47 \pm 41,34$ & $2,48 \pm 2,32$ & $95,55 \pm 3,37$ \\
\hline
\end{tabular}

La restauración, a partir de campos de arroz que se dejen de cultivar, de humedales en los márgenes de la laguna puede tener la doble finalidad de restaurar áreas de un habitat disminuido en extensión durante las últimas décadas y de "filtro" para la retención de nutrientes. El incremento de las áreas cubiertas de vegetación helofítica y/o halófila en el entorno de las lagunas costeras supone la recuperación de una situación anterior que se había alterado por los intereses de producción agrícola. Esto supuso, en el caso de la zona costera de la laguna La Tancada una disminución notable de la diversidad de habitats (Fig. 6). A modo de simulación, una restauración consistente en la recuperación de humedales con vegetación helofítica a partir de arrozales en una franja de 100 metros de anchura en el margen de la laguna supondría una recuperación de la distribución de áreas ocupadas por distintos tipos de ecosistemas hasta una situación similar a la de los años setenta. De esta manera se disminuiría la homogeneización ambiental creada por la extensión de los arrozales y, consecuentemente, se recuperaría la diversidad paisajística existente en los años 70.

\section{DISCUSIÓN}

La variación temporal de dos características tan simples como la temperatura y la salinidad, o conductividad, del agua en ecosistemas acuáticos costeros es indicadora del régimen hidrológico del agua. Es una información necesaria para conocer la importancia relativa de los aportes de agua dulce y de agua marina, y puede ser esencial para reconocer la importancia de la evaporación del agua frente a la mezcla con aguas de distinta salinidad. En zonas de clima mediterráneo, y bajo régimen hidrico no alterado, es habitual que aumente la salinidad, incluso hasta condiciones de hipersalinidad, en los periodos más calurosos que suelen coincidir con una menor pluviometría. La desviación de este patrón, como es el caso de las lagunas del Delta del Ebro (Comín et al., 1987) y en particular de la Tancada (Fig. 2) muestra la existencia de una perturbación de los flujos de agua. Para una misma masa de agua costera, el cambio en la distribución de los valores de temperatura y salinidad obtenidos en periodos de tiempo equivalentes meteorológicamente, como en la Tancada en años diferentes, muestra la variación del régimen hidrológico y da idea de la intensidad de estos cambios.

Los cambios en las cantidades absolutas y relativas de los micronutrientes disueltos en el agua son indicadores de cambios en las condiciones biogeoquímicas, cuando tienen lugar de forma persistente durante más de un periodo favorable para la producción biológica y el reciclado de la materia orgánica. La menor renovación del agua, favorece el acúmulo de la producción biológica interna al sistema y la descomposición de esta liberando nutrientes que, en parte, se acumulan en el sedimento y en el agua.

El reparto de la producción primaria entre fitoplancton, macroalgas, y macrófitos enraizados sumergidos (otros grupos como el microfito- 
bentos pueden ser importantes en ambientes con luz y no sometidos a agitación ni intensa ni frecuente) responde al tiempo de adaptación de estos productores primarios a las condiciones ambientales. El fitoplancton puede existir siempre, y más cuantos más micronutrientes tenga disponibles. Los macrófitos enraizados sumergidos tienen periodos de desarrollo individual y poblacional más largos y la presencia de los otros productores primarios representa una competición que puede ser excluyente si les limita la luz o los nutrientes. Las macroalgas tienen facilidad para crecer rápidamente en condiciones favorables de salinidad y nutrientes y de almacenar estos en sus tejidos a modo de reserva, para utilizar en periodos de escasez en el agua libre, durante los cuales se imponen a los otros grupos, aunque requieren de cierta estabi- lidad en la columna de agua para su persistencia ya que no poseen órganos de fijación al sustrato.

Los cambios a lo largo del tiempo en las características que se acaban de citar, pueden servir de indicadores de cambios en las condiciones generales de ecosistemas acuáticos costeros, siempre que se disponga de datos de estos cambios obtenidos periódicamente.

Para mejorar las condiciones ambientales de este tipo de ambientes es conveniente disponer de la posibilidad de actuar sobre los flujos de agua o, cuando menos, de regular los aportes de nutrientes a través de ellos. La disposición de "filtros" vegetales alrededor de estos ecosistemas, puede conseguir la doble finalidad de disminuir la entrada de nutrientes y de restaurar habitats naturales que han disminuido notablemente de forma generalizada. En el caso del nitrógeno, y sobre todo

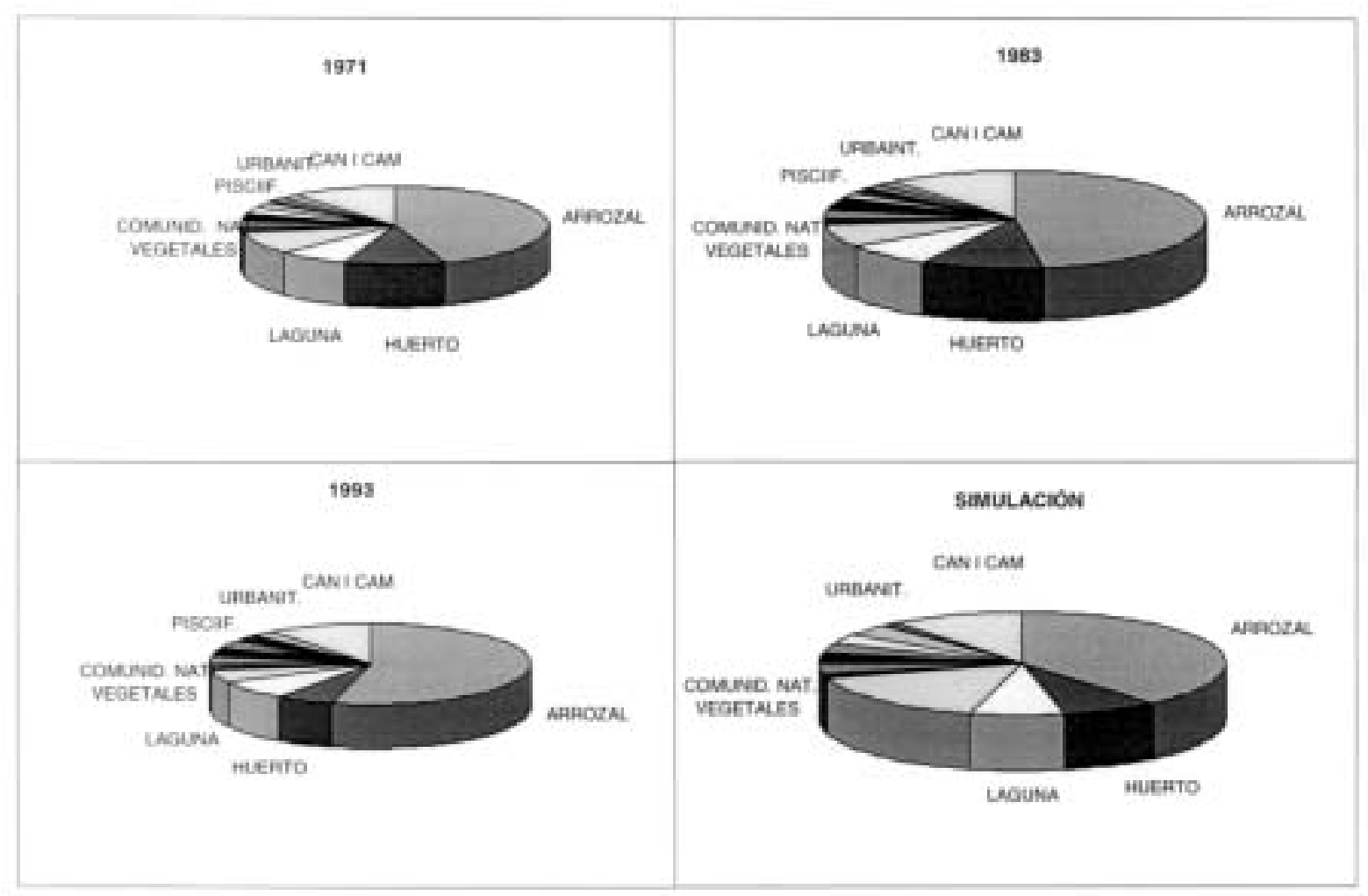

Figura 6. Variación de la diversidad de habitats en la zona costera de la laguna La Tancada durante las últimas décadas y simulación (ver texto). Variation in coastal habitat diversity of the Tancada lagoon in the past decades and simulation (see text for details). 
por el efecto de la desnitrificación (Astorga et al., 1996) puede llegar a evitarse hasta el $99 \%$ de los aportes. En el caso del fósforo, es mucho menos efectivo y se requiere una combinación de circulación del agua y crecimiento vegetal para conseguir buenos resultados.

En este sentido, la mejor herramienta a considerar para establecer estrategias de gestión de ambientes costeros es analizar su dinámica espacial a medio y largo plazo y tomar decisiones sobre el uso del territorio en base a los objetivos que se deseen, comparando lo esperado según la tendencia que tengan los usos del territorio con las previsiones según otros usos. En el caso de la zona de la Tancada en el Delta del Ebro, y practicamente en todo el litoral peninsular, este tipo de análisis nos muestra que se sigue perdiendo diversidad paisajística o de habitat y que una buena estrategia para revertir esta tendencia es restaurar hacia habitats naturales ambientes degradados o usados hoy día para finalidades que la sociedad demande a la baja. La recuperación de orlas de vegetación alrededor de zonas húmedas sería un objetivo deseable en una estrategia de este tipo.

\section{AGRADECIMIENTOS}

Este trabajo se realizó dentro del proyecto EUREED (CE-ENV4-CT95-0147) y recibió ayuda financiera del proyecto CICYT-AMB920480. Se agradecen los permisos concedidos por el Parque Natural del Delta del Ebro-Generalitat de Catalunya y la colaboración desinteresada de numerosos estudiantes en las tareas de campo.

\section{REFERENCIAS}

APHA, AWWA and WPCF 1992. Métodos normalizados para el análisis de aguas potables y residuales, ( $1^{a}$ Ed.). Ediciones Díaz de Santos, S.A., Madrid.

ASTORGA, V., E. NOVELLA \& F. A. COMÍN. 1996. $\mathrm{N}_{2} \mathrm{O}$ production in freshwater wetlands with and without added nitrate. In: Progress in Nitrogen Cycling Studies O. van Cleemput (ed.): 517-522. Kluwer Academic Publishers, Dordrecht.
COMÍN, F. A., M. MENÉNDEZ \& E. FORÉS. 1987. Salinidad y nutrientes en las lagunas costeras del delta del Ebro. Limnetica, 3:1-8.

COMÍN, F.A., M. MENÉNDEZ \& J.A. LUCENA. 1990. Proposals for macrophyte restoration in eutrophic coastal lagoons. Hydrobiologia, 200/201: 427-436.

COMÍN, F. A., M. MENÉNDEZ \& M. MARTÍN. 1991. Short-term effects of decreasing water discharge on the chemical and biological characteristics of eutrophic coastal lagoons. Mem. Ist. Ital. Idrobiol., 48: 9-22.

COMÍN, F. A., M. MARTÍN, M. MENÉNDEZ, J. A. ROMERO \& J.A. HERRERA-SILVEIRA. 1995. Integrated management of a coastal lagoon in the Ebro Delta. Proceedings MEDCOAST'95. Tarragona (Spain): 883-895.

COMÍN, F. A., J.A. ROMERO, V. ASTORGA \& C. GARCÍA. 1997. Nitrogen removal and cycling in restored wetlands used as filters of nutrients for agricultural runoff. Wat. Sci. Tech., 35(5): 255-261.

COMÍN, F. A., E. FORÉS \& M. MENÉNDEZ. 1998a. Nitrogen and phosphorus removal from agricultural sewage by wetlands under constrasting hydrologic regimes. Oecologia Aquatica, 11: 11-22.

COMIN, F. A., J. A. ROMERO \& C. GARCIA. 1998b. Removal of nitrogen and phosphorus from agricultural sewage in two restored wetlands. Verh. Internat. Verein. Limnol., 26: 1316-1320.

KOROLEFF, F. 1983. Total and organic nitrogen, In: Methods of Seawater Analysis. K. Grasshoff, M. Ehrhardt \& K. Kremling (eds): 162-173. Verlag Chemie, Weinheim.

MENÉNDEZ, M. \& F. A. COMÍN. 1989. Seasonal patterns of biomass variation of Ruppia cirrhosa (Petagna) Grande and Potamogeton pectinatus L. in a coastal lagoon. Scientia Marina, 53: 633-638.

STRICKLAND, J. D. H. \& T. R. PARSONS. 1968. A Practical Handbook of Seawater Analysis. Bull. Fish. Res. Bd. Can., 167: 311 pp. 